\title{
Isomeric Differentiation of Green Tea Catechins Using Gas-Phase Hydrogen/Deuterium Exchange Reactions
}

\author{
Emily D. Niemeyer ${ }^{\mathrm{a}}$ and Jennifer S. Brodbelt ${ }^{\mathrm{b}}$ \\ ${ }^{a}$ Department of Chemistry and Biochemistry, Southwestern University, Georgetown, Texas, USA \\ ${ }^{\mathrm{b}}$ Department of Chemistry and Biochemistry, University of Texas at Austin, Austin, Texas, USA
}

\begin{abstract}
Hydrogen/deuterium exchange reactions in a quadrupole ion trap mass spectrometer are used to differentiate galloylated catechin stereoisomers (catechin gallate and epicatechin gallate; gallocatechin gallate and epigallocatechin gallate) and the nongalloylated analogs (catechin and epicatechin, gallocatechin and epigallocatechin). Significant differences in the hydrogen/ deuterium exchange behavior of the four pairs of deprotonated catechin stereoisomers are observed upon reaction with $\mathrm{D}_{2} \mathrm{O}$. Interestingly, the nongalloylated catechins undergo $\mathrm{H} / \mathrm{D}$ exchange to a much greater extent than the galloylated species, incorporating deuterium at both aromatic/allylic and active phenolic sites. Nongalloylated catechin isomers are virtually indistinguishable by their $\mathrm{H} / \mathrm{D}$ exchange kinetics over a wide range of reaction times $(0.05$ to $10 \mathrm{~s}$ ). Our experimental results are explained using high-level ab initio calculations to elucidate the subtle structural variations in the catechin stereoisomers that lead to their differing H/D exchange kinetics. (J Am Soc Mass Spectrom 2007, 18, 1749-1759) (C 2007 American Society for Mass Spectrometry
\end{abstract}

$\mathrm{T}$ andem mass spectrometry has become a powerful and widely-applied technique for isomeric differentiation of a wide variety of compounds ranging from peptides and biomolecules [1-4] to flavonoids [5, 6] and steroids [7]. Typically, differences in mass spectral fragmentation pathways for isomeric molecules are elucidated using collisionally activated dissociation (CAD) methods, which may be further enhanced by metal complexation strategies. Opportunities for successful differentiation of isomeric compounds may be significantly expanded, however, by coupling traditional tandem mass spectrometry with ion-molecule reaction techniques [8]. Many ion-molecule reactions are highly sensitive to molecular structure and can therefore be exploited to derivatize isomers in a way that may enhance differences in mass spectral fragmentation patterns, reflect inherent conformational variations, and reveal dissimilarities in the selectivities and efficiencies of molecular reactions.

In particular, hydrogen/deuterium (H/D) exchange reactions are a very sensitive probe of molecular structure, especially conformation [8-31]. Because H/D exchange is a low-energy reaction, it can be used to evaluate the intrinsic structures of gas-phase ions, unlike the more energetic CAD methods, which impart significant internal energy and may cause rearrangements and substantial disruption of intramolecular in-

Address reprint requests to Dr. E. D. Niemeyer, Department of Chemistry and Biochemistry, Southwestern University, 315 Fondren-Jones Science Hall, Georgetown, TX 78626, USA. E-mail: niemeyee@southwestern.edu teractions [8]. We have been interested in exploring the use of $\mathrm{H} / \mathrm{D}$ exchange reactions for isomer differentiation and developing correlations with conformational effects for several years $[9,10]$, and we have recently focused our attention on the H/D exchange reactions of green tea catechin stereoisomers because of the difficulty in distinguishing these compounds using traditional tandem mass spectrometric methods.

All catechins share a general flavan-3-ol structure and are found naturally in a number of plant-derived products, but they occur in particularly high concentration in green tea. The most abundant catechins in green tea exist as four pairs of stereoisomers: catechin and epicatechin; epigallocatechin (EGC) and gallocatechin (GC); catechin gallate (CG) and epicatechin gallate (ECG); and epigallocatechin gallate (EGCG) and gallocatechin gallate (GCG) (see Figure 1 for structures). Green tea catechins have been thoroughly studied in vitro, and are known to have potent antioxidant activity $[32,33]$ and have been shown to inhibit carcinogenesis, mutagenesis, and tumorigenesis [34-36]. Green tea consumption has been correlated with inhibition of certain types of cancer (primarily of the digestive system) [37, $38]$ and the prevention of heart disease [37, 39].

Like many flavonoids, the bioavailability and bioefficacy of the catechin isomers differ greatly depending on their molecular structure [40, 41]. Differences in catechin structure are known to directly affect the absorption, metabolism, and elimination of these compounds in humans [35, 42]. For example, EGCG, the most prevalent catechin in green tea and arguably the 

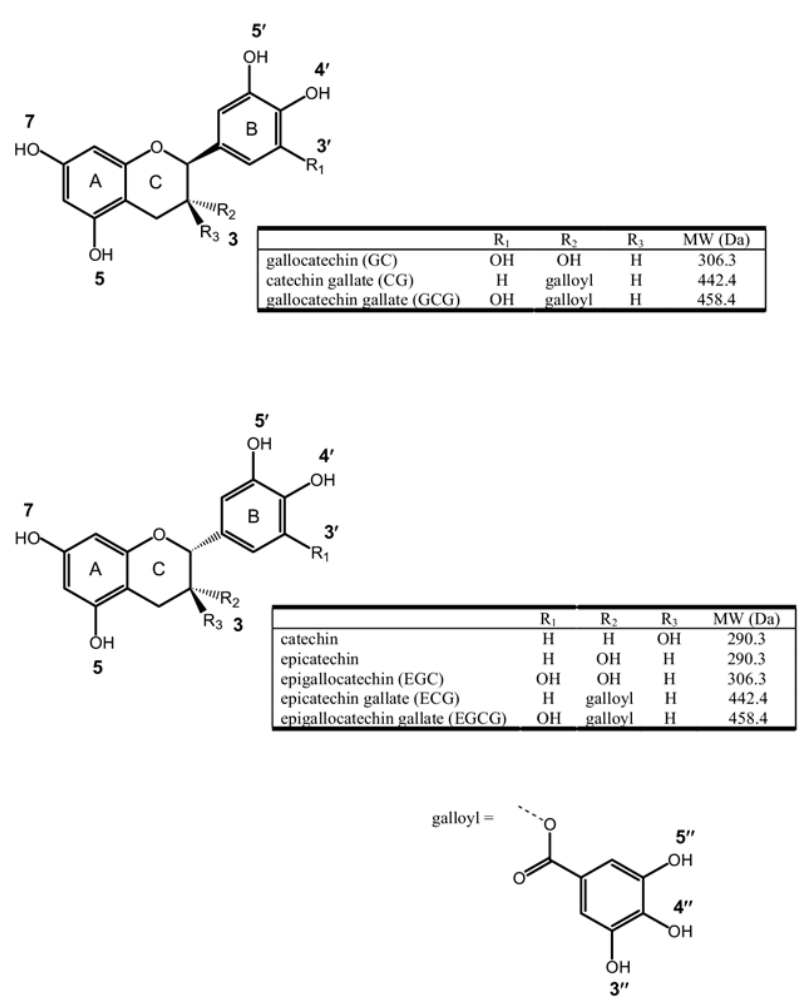

Figure 1. Chemical structures and molecular weights (MW) of the green tea catechins used in this study.

most studied, is preferentially excreted in bile while other nongalloylated catechins are rapidly eliminated in urine [35]. The galloyl moiety has also been shown to increase radical scavenging capabilities, with EGCG and ECG having significantly larger Trolox equivalent antioxidant activity (TEAC) values than the other catechins present in green tea [37]. Accurate structural determination is therefore extremely important in developing a more complete understanding of the biological properties and activities of green tea catechins.

Mass spectrometry (MS) is a prevalent technique for the elucidation of flavonoid structure [43-45], and electrospray ionization (ESI) and atmospheric pressure chemical ionization (APCI) MS methods are most often used for the analysis of catechins. Green tea catechins have been previously identified by direct infusion ESI-MS [46] and tandem MS (MS/MS) [47, 48], but these techniques fail to distinguish the structures of catechin stereoisomers. Only by pairing gas $[49,50]$ or liquid [51-54] chromatography with mass spectrometric detection can two stereoisomers such as catechin and epicatechin (molecular masses $=290$ Da; see Figure 1) be differentiated by mass spectral analysis.

In the present study, we explore the H/D exchange reactions of four sets of isomeric catechins, with an emphasis on examining the impact of stereoisomerism on the extent and kinetics of $H / D$ exchange. $H / D$ exchange is a powerful technique for gaining structural information about stereoisomers, and it has been previously used to differentiate isomeric flavonoid aglycones and glycosides by tandem MS [9] and methylated flavonoid derivatives by chemical ionization MS [31].

We have used a quadrupole ion trap (QIT) mass spectrometer to monitor gas-phase $H / D$ exchange reactions between deuterium oxide $\left(\mathrm{D}_{2} \mathrm{O}\right)$ and eight green tea catechins, including four nongalloylated (catechin, epicatechin, EGC, and GC) and four galloylated (CG, ECG, EGCG, and GCG) stereoisomers. Our experimental results are explained using ab initio calculations that elucidate the subtle variations in the catechin stereoisomers that affect their overall H/D exchange kinetics.

\section{Experimental}

\section{Chemicals and Reagents}

(+)-Catechin hydrate, (-)-epicatechin, (-)-gallocatechin, (-)-epigallocatechin, (-)-gallocatechin gallate, (-)-epigallocatechin gallate, $(-)$-catechin gallate and (-)-epicatechin gallate were purchased from Sigma-Aldrich (Milwaukee, WI). Deuterium oxide $\left(\mathrm{D}_{2} \mathrm{O}\right)$ was obtained from Cambridge Isotope Laboratories (Andover, MA) and HPLC-grade methanol $(\mathrm{MeOH})$ was purchased from Fisher Scientific (Fair Lawn, NJ). All chemicals and reagents were used without further purification.

Stock solutions of each catechin stereoisomer in $\mathrm{MeOH}$ were prepared at concentrations ranging from 1 to $3 \mathrm{mM}$ and stored at $4{ }^{\circ} \mathrm{C}$ until use. Dilute working solutions of the catechin stereoisomers in $\mathrm{MeOH}(40-$ $100 \mu \mathrm{M})$ were then prepared from the stock standards immediately before mass spectral analysis.

\section{H/D Exchange Conditions}

A Hitachi (Tokyo, Japan) 3DQ quadrupole ion trap mass spectrometer equipped with an electrospray ionization (ESI) source was used for all H/D exchange experiments. Catechin isomer solutions were introduced into the ESI source by direct infusion using a Harvard Apparatus syringe pump (Holliston, MA) set at a rate of $5 \mu \mathrm{L} / \mathrm{min}$ with the $\mathrm{MeOH}$ makeup flow at 80 $\mu \mathrm{L} / \mathrm{min}$. The ESI probe, focus, and drift voltages were individually optimized for each pair of catechin stereoisomers. The following instrumental parameters were held constant for all experiments: nitrogen sheath gas = $2 \mathrm{~kg} / \mathrm{cm}^{2}$; helium damping gas $=2 \mathrm{~kg} / \mathrm{cm}^{2}$; assistant gas heater $=200{ }^{\circ} \mathrm{C}$; desolvation temperature $=150{ }^{\circ} \mathrm{C}$; and aperture temperature $=150{ }^{\circ} \mathrm{C}$.

Before an $\mathrm{H} / \mathrm{D}$ exchange experiment, the catechin of interest was first detected in its deprotonated form (negative ion mode) with the helium buffer gas pressure at nominally $3.5 \times 10^{-5}$ torr, as read from the ion pressure gauge. The exchange reagent, deuterium oxide, was then introduced into the ion trap using a house-designed leak assembly that has been previously described in detail [9]. The nominal $\mathrm{D}_{2} \mathrm{O}$ pressure in the vacuum chamber was $3.5 \times 10^{-4}$ torr and the reaction/ exchange time with the catechin isomer of interest was varied between 0.05 and $10 \mathrm{~s}$. For all exchange reactions, 
the ion accumulation time was $200 \mathrm{~ms}$ and the isolation time was set at $20 \mathrm{~ms}$.

All H/D exchange data were corrected for isotopic contributions and normalized before data fitting. H/D exchange rate constants and kinetic plots were determined using KinFit [55].

\section{Molecular Modeling and Acidity Calculations}

Low-energy conformations were determined for catechins in this study and their respective anions either by manual systematic searching at the semi-empirical (PM3) level (catechin, epicatechin) or by conformational sampling using a Monte Carlo method with the MMFF force-field (GC, EGC, CG, ECG) in the Spartan '04 Essential Edition software package (Wavefunction, Irvine, CA) [56]. All structures were then further optimized using ab initio methods at the HF/6-31G + (d,p) level using the Gaussian 03 software package [57]. Vibrational frequencies were computed at the same level of theory and scaled by a factor of 0.9153 to determine the corrected zero-point energies $\left(\mathrm{ZPE}_{\mathrm{C}}\right)$ [58]. [Currently no reported zero-point vibrational energy scaling factor exists in the literature for the HF/6-31G + $(d, p)$ level of theory. Therefore, we used the scaling factor developed for HF/6-31 + G(d), a value of 0.9153 [58]. We anticipate this has no significant impact on our calculated gas phase acidities because of both the inherent uncertainties associated with the determination of scaling factors [59] and due to the fact that the zeropoint correction has only a small contribution to our calculated electronic energy $\left(\mathrm{E}_{\text {elec }}\right)$.] Single-point energies $\left(E_{0}\right)$ were then calculated for each of the optimized neutral (HA) and anionic $\left(\mathrm{A}^{-}\right)$catechins at the MP2/6$311+\mathrm{G}(\mathrm{d}, \mathrm{p})$ level. The gas-phase acidity $\left(\Delta \mathrm{H}_{\text {acidity }}\right)$ was finally computed for each catechin as the difference between the total energy $\left(\mathrm{E}_{\text {elec }}\right)$ of the respective anion $\left(\mathrm{E}_{\text {elec,A- }}=\mathrm{E}_{0, \mathrm{~A}_{-}}+\mathrm{ZPE}_{\mathrm{C}, \mathrm{A}-}\right)$ and neutral species $\left(\mathrm{E}_{\text {elec,HA }}\right.$ $\left.=\mathrm{E}_{0, \mathrm{HA}}+\mathrm{ZPE} \mathrm{E}_{\mathrm{C}, \mathrm{HA}}\right)$. Relative acidities were calculated as the difference between the absolute acidity and the lowest calculated anion acidity for each compound. Our methodology was closely based on several previous theoretical studies of the gas-phase acidities of flavonoids $[9,60,61]$ to balance accuracy with our available computational resources.

\section{Results and Discussion}

All of the green tea catechins used in the present study form abundant deprotonated molecular ions upon electrospray ionization and were therefore analyzed in the negative ion mode. Previous research has shown that, in general, the negative ion mode is preferable for the ESI-MS analysis of flavonoids because it affords better sensitivity due to the ease of deprotonating acidic flavonoid hydroxyl groups [62, 63].

$\mathrm{H} / \mathrm{D}$ exchange reactions were completed with all catechin isomers using deuterated water $\left(\mathrm{D}_{2} \mathrm{O}\right)$ as the exchange reagent. Deuterium oxide was chosen because

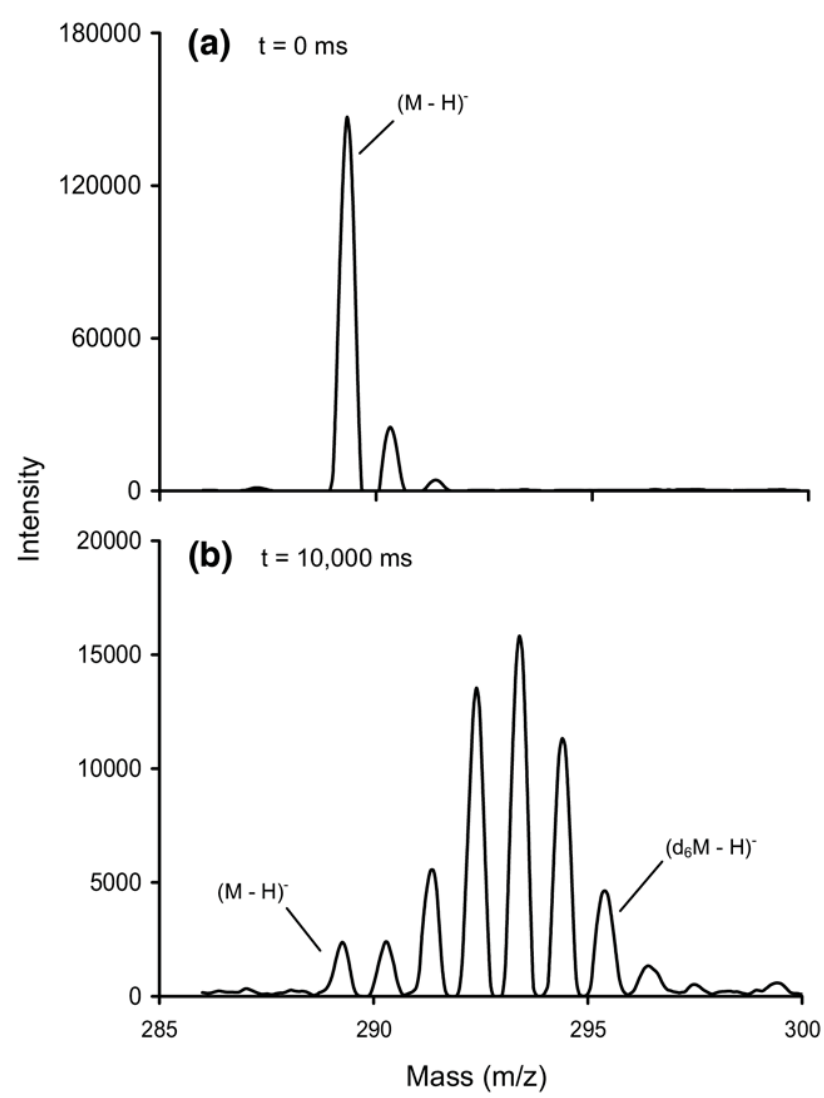

Figure 2. Mass spectra of the deprotonated catechin molecular ion, $(\mathrm{M}-\mathrm{H})^{-}$, before $\mathrm{H} / \mathrm{D}$ exchange (a) and after 10,000 ms reaction time with $\mathrm{D}_{2} \mathrm{O}(\mathbf{b})$.

a previous comparison of the reactivity of several common $\mathrm{H} / \mathrm{D}$ exchange reagents $\left(\mathrm{D}_{2} \mathrm{O}, \mathrm{CD}_{3} \mathrm{OD}\right.$, and $\mathrm{ND}_{3}$ ) with the flavonoids neohesperidin and rutin showed $\mathrm{D}_{2} \mathrm{O}$ to be the "most effective" exchange reagent for the model flavonoids studied [9].

\section{H/D Exchange of Nongalloylated Catechin Stereoisomers}

During a typical gas-phase H/D exchange experiment, some portion of the labile hydrogens on the analyte ion, usually those attached to nitrogen, oxygen, or sulfur atoms, may be replaced by deuterium atoms from the exchange reagent. The process by which this exchange occurs can vary, but the rate and extent of deuterium incorporation relates to specific properties of the analyte (e.g., gas-phase acidity, deprotonation/charge site, conformation) and exchange reagent (e.g., gas-phase acidity/basicity). Figure 2 shows a typical mass spectrum for the deprotonated catechin molecular ion, denoted as $(\mathrm{M}-\mathrm{H})^{-}$, before the addition of $\mathrm{H} / \mathrm{D}$ exchange reagent (Figure $2 \mathrm{a}$ ) and after reaction with deuterium oxide (Figure 2b). At 10,000 ms, the longest exchange time used in the present study, a peak for the deprotonated molecular ion remains visible but incorporation of up to six deuteriums, $\left(\mathrm{d}_{6} \mathrm{M}-\mathrm{H}\right)^{-}$, is observed. 
Table 1 presents the expected number of labile hydrogens for each of the deprotonated nongalloylated catechin isomers along with the maximum number of exchanges observed after $10 \mathrm{~s}$ reaction time. All of the nongalloylated catechin isomers have a greater number of $\mathrm{H} / \mathrm{D}$ exchanges than predicted by the number of labile hydrogens for the deprotonated molecules, implying that deuterium exchange is occurring with aromatic or allylic nonlabile hydrogens. Interestingly, deuterium exchange occurs to a significantly greater extent at nonlabile sites on gallocatechin and epigallocatechin (four nonlabile proton exchanges) than for catechin and epicatechin (two nonlabile proton exchanges), although the gallocatechins differ from the catechins only by the addition of a third hydroxyl group on the flavonoid B ring (see Figure 1). Typically, nonlabile hydrogens are exchanged with deuterium during more energetic processes, such as chemical ionization [31, 64], but are rarely observed after creation of ions by ESI. However, Reed and Kass demonstrated that gentle activation or energy accumulation during ESI can provide sufficient energy to cause exchange at nonlabile sites via a scrambling mechanism [11]. By studying a series of carboxylate ions under various instrumental conditions, the authors found that scrambling occurs via transfer of a "relatively acidic" nonlabile hydrogen to the deprotonation site followed by deuterium transfer to the new charge location from a deuterated group elsewhere on the ion.

A previous computational study utilizing semi-empirical (PM3, AM1) methods and combined density function theory (DFT) to study bond dissociation energies provides insight into the relative acidities of allylic and phenolic protons in green tea catechins [65]. Zhang and Wang calculated the bond dissociation energies (BDEs) of allylic and hydroxyl protons on epicatechin and epigallocatechin and showed that the allylic hydrogen at the carbon-2 (C2) position on the flavonoid $\mathrm{C}$ ring has a $\mathrm{BDE}$ comparable to the phenolic $3^{\prime}-\mathrm{OH}$ proton [65]. Their work confirmed an experimental and semi-empirical computational study by Kondo and coworkers, which suggested abstraction of allylic hydrogens from epicatechin and epigallocatechin plays an important role in the antioxidative effect of these compounds [66]. Both of these studies confirm the presence of relatively acidic nonphenolic hydrogens that we speculate may participate in a scrambling mechanism during $\mathrm{H} / \mathrm{D}$ exchange reactions for the nongalloylated catechins. This scrambling process could lead to deuterium incorporation at nonlabile sites by a mechanism such as that proposed in Scheme $\mathbf{1}$.

To probe further differences in the H/D exchange reactivity of the nongalloylated catechins with deuterium oxide, kinetic data were collected and are summarized in Table 1. All rate constants were extracted from kinetic plots such as the one shown for catechin in Figure 3. Over the exchange time studied, the relative abundance of nondeuterated catechin, $\mathrm{D}(0)$, decreases

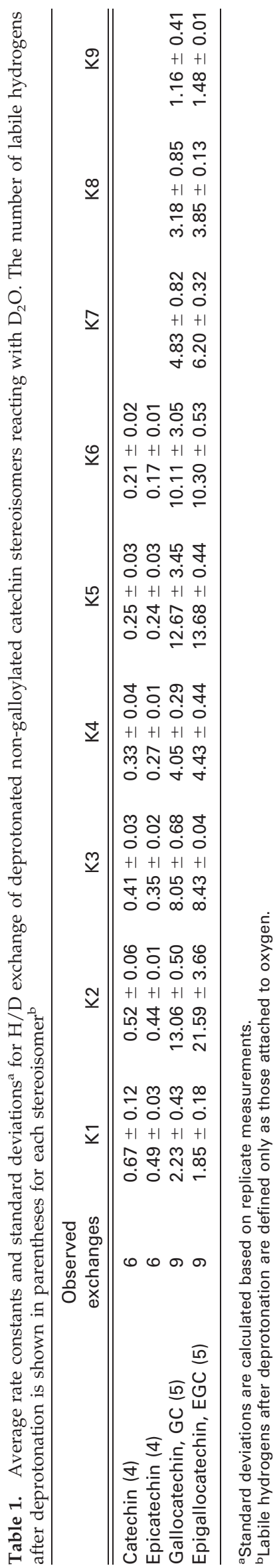




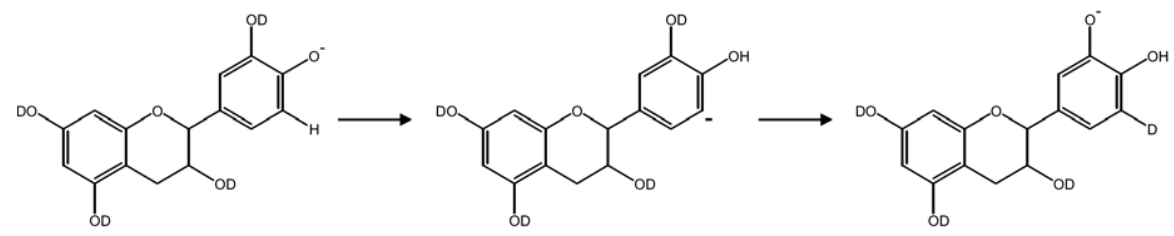

Scheme 1. Proposed scrambling mechanism allowing deuterium incorporation at nonlabile sites on a flavonoid such as catechin or epicatechin. Adapted from Reed and Kass [11].

while the other deuterated species, $\mathrm{D}(1)$ through $\mathrm{D}(6)$, subsequently increase.

Stereoisomerism was found to have little effect on the reaction kinetics of the nongalloylated catechins with deuterium oxide. Catechin and epicatechin incorporate deuterium to the same extent (six exchanges) with similar rate constants for each deuterium exchange. Gallocatechin and epigallocatechin also exchange the same number of deuteriums (nine) with comparable individual rate constants.

However, comparing the H/D exchange kinetics of catechin/epicatechin with gallocatechin/epigallocatechin reveals significant differences not only in the extent of deuterium incorporation, as discussed above, but also in the apparent rate constants. Although it is difficult to make a quantitative comparison of rate constants since the nongalloylated catechins have multiple sites for deuterium exchange, it is evident when comparing the gallocatechins to the catechins that the addition of a single hydroxyl group on the flavonoid B ring (see Figure 1), leads to substantially faster rates of deuterium incorporation. This result echoes previous studies that have shown that the addition of adjacent hydroxyl groups on the $\mathrm{B}$ ring facilitates fast $\mathrm{H} / \mathrm{D}$ exchange in flavonoids such as rutin and astragalin [9]. For the gallocatechins, the presence of three adjacent hydroxyl groups on the flavonoid B ring may also help

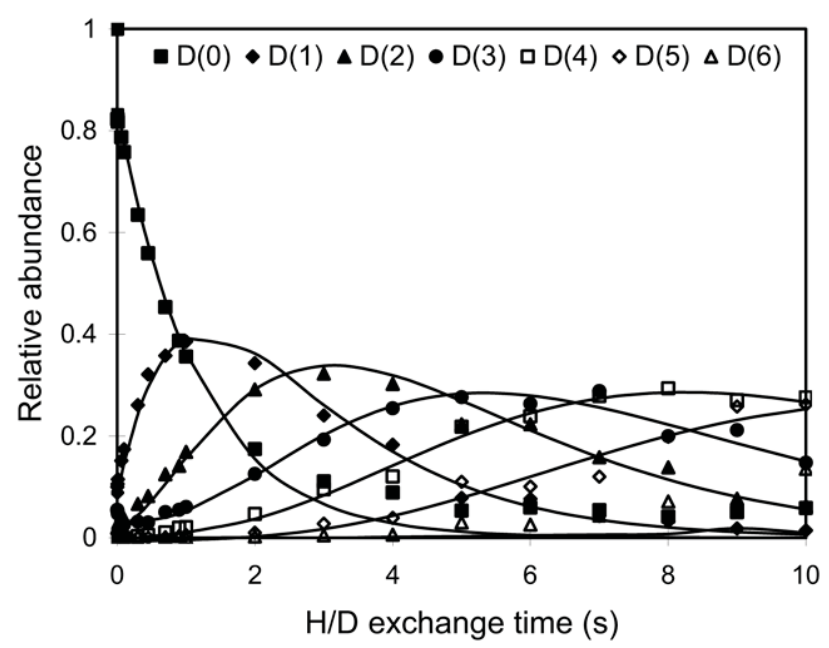

Figure 3. Kinetic plot of the H/D exchange reaction of deprotonated catechin with $\mathrm{D}_{2} \mathrm{O}$. Individual points represent experimentally-determined values and lines are determined by KinFit [55]. $\mathrm{D}(0)$ represents the initial parent ion and $\mathrm{D}(x)$ represents ions incorporating from one to $x$ deuteriums. to explain the substantially greater extent of deuterium incorporation for these molecules (nine exchanges) compared with the catechins (six exchanges). Closer inspection of the rate constants for the gallocatechins reveals that the first deuterium exchange (K1) is significantly slower than subsequent reactions and the second (K2) and fifth (K5) deuterium exchanges have the fastest overall reaction rates. Such an unusual H/D exchange pattern may indicate that deuterium exchanges are simultaneously occurring at multiple charge sites on the gallocatechins [12]. Examination of the rate constants for the catechins, however, shows that the first deuterium exchange (K1) is the fastest with the reaction rate decreasing for subsequent exchanges which is indicative of a typical sequential deuterium exchange pattern. Therefore, the presence of three hydroxyl groups on the gallocatechin/epigallocatechin B ring not only facilitates faster $\mathrm{H} / \mathrm{D}$ exchange rates but also provides alternate charge sites in close proximity which likely activates simultaneous deuterium exchanges at several locations on the molecule causing extensive deuterium incorporation.

\section{H/D Exchange of Galloylated Catechin Stereoisomers}

Kinetic data for the reaction of the galloylated catechins with deuterium oxide is compiled in Table 2. All of the galloylated catechins undergo extensive exchange but only incorporate deuterium to the extent expected by the number of labile hydrogens on each ion after deprotonation. Thus, in contrast to the nongalloylated catechins, there is no evidence that the galloylated catechins incorporate deuterium at nonlabile sites via a scrambling mechanism. Presumably the galloylated catechins react with deuterium oxide via a typical relay mechanism [12], similar to the H/D exchange observed for other flavonoids with $\mathrm{D}_{2} \mathrm{O}$ [9].

Inspection of the data in Table 2 also reveals that stereoisomerism has a considerable impact on the H/D exchange kinetics of the galloylated catechins. Although catechin gallate and epicatechin gallate exhibit the same maximum number of observed exchanges (six), the magnitude of the individual rate constants differs significantly for these isomers. In particular, the rate constants for the incorporation of the fifth and sixth deuteriums, K5 and K6, are substantially larger (by a factor of ten to twenty) for catechin gallate than epicatechin gallate. It is also interesting to note that for 


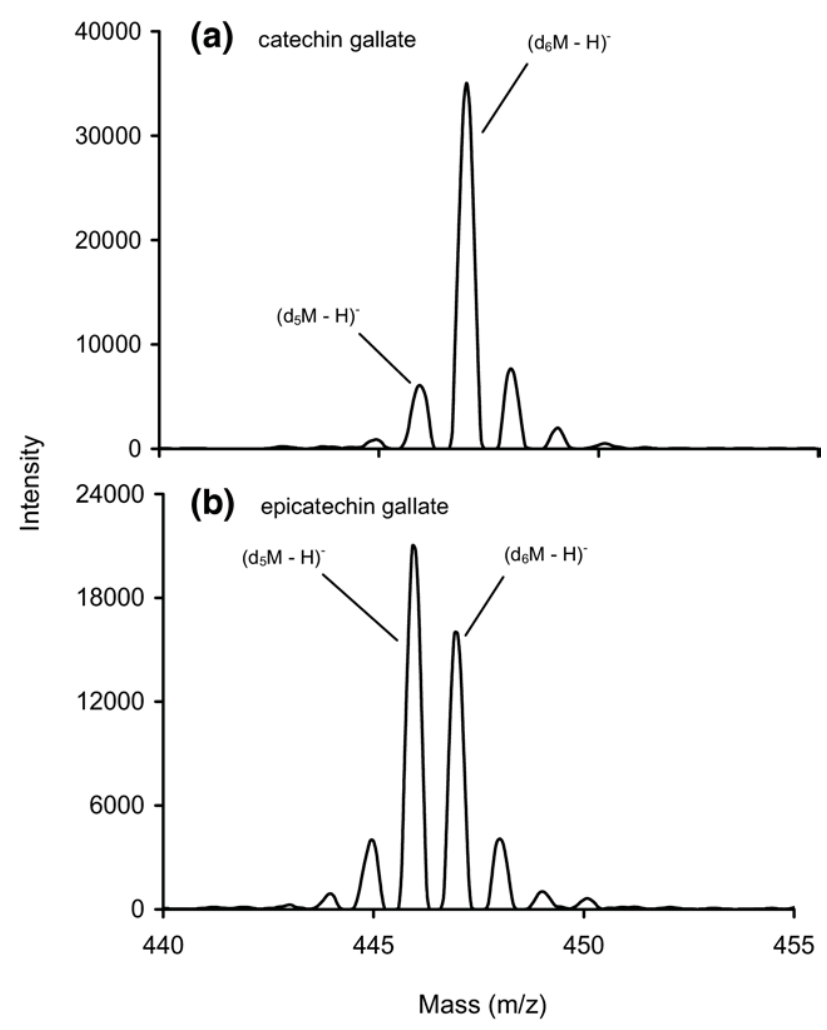

Figure 4. H/D exchange reaction of catechin gallate (a) and epicatechin gallate $(\mathbf{b})$ with $\mathrm{D}_{2} \mathrm{O}$ after $10,000 \mathrm{~ms}$ reaction time.

catechin gallate, the reaction rate for incorporation of the fifth deuterium (K5) is significantly greater than $\mathrm{K} 2$, $\mathrm{K} 3$, and K4. Although this same pattern of deuterium exchange was observed for the gallocatechins, it does not occur for any of the other galloylated catechins, implying that catechin gallate may have a pattern of deuterium exchange unique to the galloylated stereoisomers, with simultaneous exchanges occurring at multiple charge sites.

The differences in rates of deuterium incorporation for catechin gallate and epicatechin gallate lead directly to observable variations in the mass spectra, particularly at longer exchange times. For example, Figure 4 shows typical H/D exchange data for catechin gallate (Figure 4a) and epicatechin gallate (Figure 4b) after $10,000 \mathrm{~ms}$. The base peak for catechin gallate corresponds to the species with six incorporated deuteriums, $\left(\mathrm{d}_{6} \mathrm{M}-\mathrm{H}\right)^{-}$, whereas the base peak for epicatechin gallate incorporates five deuteriums, $\left(\mathrm{d}_{5} \mathrm{M}-\mathrm{H}\right)^{-}$.

When the stereoisomers gallocatechin gallate and epigallocatechin gallate react with deuterium oxide, they also have the same number of observed exchanges (seven). However, comparison of their H/D exchange kinetics shows that the first rate constants for deuterium exchange (K1, K2, and $\mathrm{K} 3$ ) are significantly larger for gallocatechin gallate than for epigallocatechin gallate although the last three exchanges (K4, K5, and K6) are statistically indistinguishable. All of the galloylated catechin stereoisomers studied, which differ only in the 
Table 3. Calculated gas-phase absolute and relative acidities for catechin, epicatechin, gallocatechin (GC), and epigallocatechin (EGC)

\begin{tabular}{|c|c|c|c|c|}
\hline \multirow[b]{2}{*}{$\mathrm{O}^{-}$position } & \multicolumn{2}{|c|}{ Acidity $(\mathrm{kcal} / \mathrm{mol})^{\mathrm{a}}$} & \multicolumn{2}{|c|}{ Relative acidity ${ }^{b}$} \\
\hline & Catechin & Epicatechin & Catechin & Epicatechin \\
\hline 3 & 368.6 & 370.6 & 27.3 & 29.7 \\
\hline 5 & 345.8 & 343.6 & 4.5 & 2.8 \\
\hline 7 & 350.0 & 348.0 & 8.7 & 7.2 \\
\hline $3^{\prime}$ & 341.5 & 340.8 & 0.2 & 0.0 \\
\hline $4^{\prime}$ & 341.3 & 341.8 & 0.0 & 1.0 \\
\hline & Gallocatechin & Epigallocatechin & Gallocatechin & Epigallocatechin \\
\hline 3 & 369.1 & 366.7 & 33.2 & 31.1 \\
\hline 5 & 346.8 & 344.1 & 10.9 & 8.5 \\
\hline 7 & 350.5 & 348.4 & 14.5 & 12.8 \\
\hline $3^{\prime}$ & 339.5 & 338.8 & 3.5 & 3.3 \\
\hline $4^{\prime}$ & 335.9 & 335.6 & 0.0 & 0.0 \\
\hline $5^{\prime}$ & 339.4 & 339.6 & 3.5 & 4.0 \\
\hline
\end{tabular}

${ }^{a}$ All gas-phase acidities $\left(\Delta \mathrm{H}_{\text {acidity }}\right)$ are computed using the MP2/6-311 $+\mathrm{G}(\mathrm{d}, \mathrm{p})$ method as the difference between the total energy $\left(\mathrm{E}_{\text {elec }}\right)$ of the respective anion $\left(\mathrm{E}_{\text {elec,A- }}=\mathrm{E}_{0, \mathrm{~A}_{-}}+\mathrm{ZPE}_{\mathrm{C}, \mathrm{A}-}\right)$ and neutral species $\left(\mathrm{E}_{\mathrm{elec}, \mathrm{HA}}=\mathrm{E}_{\mathrm{O}, \mathrm{HA}}+\mathrm{ZPE} \mathrm{E}_{\mathrm{C}, \mathrm{HA}}\right)$ where $\mathrm{ZPE} \mathrm{E}_{\mathrm{C}}$ is the corrected zero-point energy calculated at the $\mathrm{HF} / 6-31 \mathrm{G}+(\mathrm{d}, \mathrm{p})$ level.

${ }^{b}$ Relative acidity is calculated as the difference between the absolute acidity and the lowest calculated anion acidity for each catechin.

chirality of the $\mathrm{C} 2$ carbon on the flavonoid $\mathrm{C}$ ring, have distinctive $\mathrm{H} / \mathrm{D}$ exchange kinetics. It is interesting to note that, in general, $\mathrm{S}$ chirality of the $\mathrm{C} 2$ carbon (CG, GCG) correlates with faster H/D exchange than $R$ (ECG, EGCG).

Comparing the H/D exchange kinetics of catechin gallate/epicatechin gallate with gallocatechin gallate/ epigallocatechin gallate further confirms that the addition of a hydroxyl group on the flavonoid B ring facilitates fast H/D exchange kinetics. Clearly, the individual $\mathrm{H} / \mathrm{D}$ exchange rate constants of gallocatechin gallate are generally larger than those of catechin gallate and the same pattern is observed for epigallocatechin gallate and epicatechin gallate.

\section{Gas-Phase Acidity Calculations for Nongalloylated and Galloylated Catechin Stereoisomers}

To rationalize these H/D exchange results, we performed high level ab initio calculations to predict the probable deprotonation sites, low-energy conformations, and gas-phase acidities for each of the nongalloylated catechin isomers as well as two of the galloylated catechin stereoisomers. [Because of their large molecular size, it was computationally impractical to complete ab initio calculations for each of the galloylated catechin stereoisomers used in our H/D exchange study. Therefore, we limited our molecular modeling study to catechin gallate and epicatechin gallate, the galloylated stereoisomers found to have the greatest differences in $\mathrm{H} / \mathrm{D}$ exchange kinetics and extent of deuterium incorporation.] Table 3 summarizes the absolute gas-phase acidities (in $\mathrm{kcal} / \mathrm{mol}$ ) and relative acidities for the nongalloylated catechins as determined at the MP2/6-311 + G(d,p) level of theory. The calculated catechin and epicatechin gasphase acidities range from 340 to $370 \mathrm{kcal} / \mathrm{mol}$ and agree with literature values determined by similar computational methods [60, 61]. Closer examination of Table 3 shows that, for catechin, the $3^{\prime}-\mathrm{OH}$ and $4^{\prime}-\mathrm{OH}$ protons have nearly the same acidity values (differing by only $0.2 \mathrm{kcal} / \mathrm{mol}$ ) with the $4^{\prime}-\mathrm{OH}$ site slightly favored for deprotonation. Our results agree directly with gas-phase acidity computations performed on catechin by Martins et al. using the B3LYP/6-311 + G(2d,2p) method [60]. Epicatechin, however, was determined to have a greater difference in calculated acidity values between the $3^{\prime}-\mathrm{OH}$ and $4^{\prime}-\mathrm{OH}$ protons $(1.0 \mathrm{kcal} / \mathrm{mol})$, with the $3^{\prime}-\mathrm{OH}$ slightly favored for deprotonation. A recent study of epicatechin at the B3LYP/6-311 ++ $\mathrm{G}^{* *}$ level also confirmed a larger gas-phase acidity for the $3{ }^{\prime}-\mathrm{OH}$ proton when compared to the $4^{\prime}-\mathrm{OH}$ group [61]. Based on the similarity in the gas-phase acidity values, however, deprotonation is likely to occur readily at the $3^{\prime}-\mathrm{OH}$ and $4^{\prime}-\mathrm{OH}$ positions for both catechin and epicatechin. The 3-OH proton is by far the least acidic for catechin and epicatechin indicating this position forms the least stable anion, as expected by its location on the nonaromatic flavonoid $C$ ring (see Figure 1). Interestingly, a direct comparison between catechin and epicatechin shows that, overall, these stereoisomers have very similar gasphase acidity values, differing by no more than 2.2 $\mathrm{kcal} / \mathrm{mol}$. Because catechin and epicatechin have comparable gas-phase acidities and probable deprotonation sites, it is not surprising that these two compounds have indistinguishable $\mathrm{H} / \mathrm{D}$ exchange kinetics. Although distinct molecular conformations may also lead to differing H/D exchange behavior and rates of deuterium incorporation, the conformational flexibility of the catechins is significant [60], making molecular conformation unlikely to affect overall $\mathrm{H} / \mathrm{D}$ exchange results. 
Table 4. Calculated gas-phase absolute and relative acidities for catechin gallate (CG) and epicatechin gallate (ECG)

\begin{tabular}{cccccc}
\hline & \multicolumn{2}{c}{ acidity $(\mathrm{kcal} / \mathrm{mol})^{\mathrm{a}}$} & & \multicolumn{2}{c}{ Relative acidity $^{\mathrm{b}}$} \\
\cline { 2 - 3 } \cline { 5 - 6 } O- position & $\begin{array}{c}\text { Catechin } \\
\text { gallate }\end{array}$ & $\begin{array}{c}\text { Epicatechin } \\
\text { gallate }\end{array}$ & & $\begin{array}{c}\text { Catechin } \\
\text { gallate }\end{array}$ & $\begin{array}{c}\text { Epicatechin } \\
\text { gallate }\end{array}$ \\
\hline \hline 5 & 339.9 & 341.4 & & 9.8 & 10.1 \\
7 & 343.3 & 344.3 & & 13.2 & 13.0 \\
$3^{\prime}$ & 330.8 & 333.2 & & 0.7 & 2.0 \\
$4^{\prime}$ & 335.5 & 340.9 & & 5.4 & 9.6 \\
$3^{\prime \prime}$ & 330.2 & 331.3 & & 0.1 & 0.0 \\
$4^{\prime \prime}$ & 331.9 & 332.9 & & 1.8 & 1.6 \\
$5^{\prime \prime}$ & 330.1 & 333.3 & & 0.0 & 2.0 \\
\hline
\end{tabular}

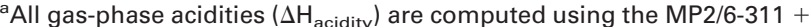
$G(d, p)$ method as the difference between the total energy $\left(E_{\text {elec }}\right)$ of the respective anion ( $\left.E_{\text {elec A- }}=E_{0 . A_{-}}+Z P E_{C, A_{-}}\right)$and neutral species $\left(E_{\text {elec,HA }}\right.$ $\left.=E_{O H A}+Z P E_{C, H A}\right)$ where $Z \mathrm{PE}_{\mathrm{C}}$ is the corrected zero-point energy calculated at the HF/6-31G + (d,p) level.

${ }^{\text {b}}$ Relative acidity is calculated as the difference between the absolute acidity and the lowest calculated anion acidity for each catechin.

The gallocatechin and epigallocatechin gas-phase acidities range from 335 to $370 \mathrm{kcal} / \mathrm{mol}$ with the $4^{\prime}-\mathrm{OH}$ proton favored for deprotonation in both compounds, although deprotonation likely occurs readily at the acidic $3^{\prime}-\mathrm{OH}$ and $5^{\prime}-\mathrm{OH}$ positions as well. For both gallocatechin and epigallocatechin, the $3-\mathrm{OH}$ proton is the least acidic, a characteristic shared among all the nongalloylated catechins. A comparison between gallocatechin and epigallocatechin shows similar values for the gas-phase acidities (differing by no more than $2.7 \mathrm{kcal} / \mathrm{mol}$ ) and nearly identical values at the most likely deprotonation sites, the $3^{\prime}-\mathrm{OH}, 4^{\prime}-\mathrm{OH}$, and $5^{\prime}-\mathrm{OH}$ positions, which differ by no greater than $0.6 \mathrm{kcal} / \mathrm{mol}$ between the two molecules. All of the nongalloylated catechins were therefore found to have similar deprotonation sites and comparable gas-phase acidities between stereoisomers. Results from our ab initio calculations correlate directly with our experimental results, showing that the $\mathrm{H} / \mathrm{D}$ exchange kinetics and rates of deuterium incorporation are not affected by stereoisomerism for the nongalloylated catechins.

Table 4 presents the acidities for catechin gallate and epicatechin gallate determined at the MP2/6-311 $+\mathrm{G}(\mathrm{d}, \mathrm{p})$ level of theory. In general, phenolic protons on the galloylated catechins are significantly more acidic than on the nongalloylated compounds (see Table 3). Calculated gas-phase acidities for catechin gallate and epicatechin gallate range from 330 to 344 $\mathrm{kcal} / \mathrm{mol}$ and the $7-\mathrm{OH}$ position was found to be the least acidic for both of the galloylated catechins. For catechin gallate, the $3^{\prime \prime}-\mathrm{OH}$ and $5 "-\mathrm{OH}$ sites on the gallate group were determined to be the most acidic, but deprotonation is also clearly favored at the $4 "-\mathrm{OH}$ and $3^{\prime}-\mathrm{OH}$ positions, which are only slightly less acidic (less than $1.8 \mathrm{kcal} / \mathrm{mol}$ ) than the most acidic hydroxyl proton. For epicatechin gallate, the $3 "-\mathrm{OH}$ group on the gallate group was found to be the most acidic, but deprotonation is also likely to readily

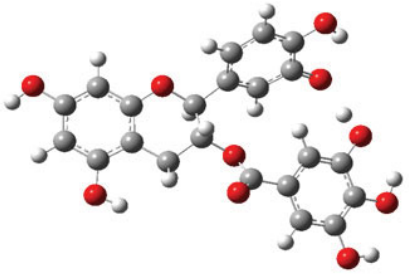

(a)

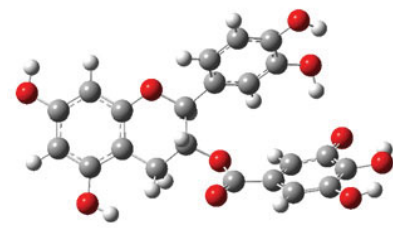

(c)

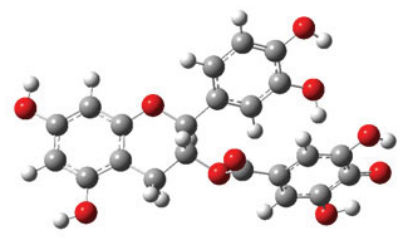

(e)

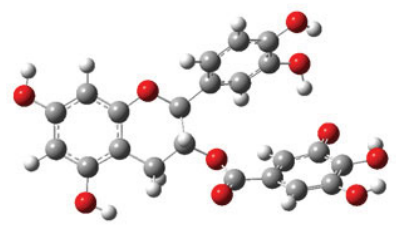

(g)

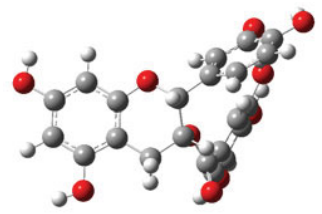

(b)

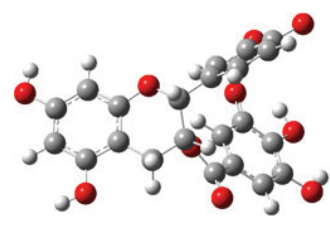

(d)

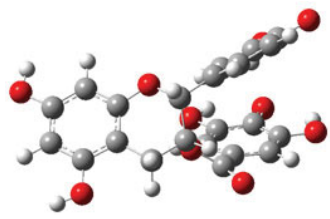

(f)

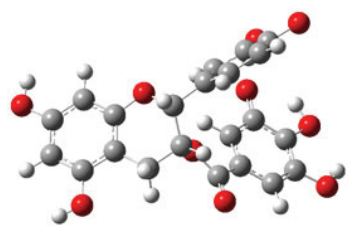

(h)
Figure 5. Lowest energy conformations of catechin gallate (a) and epicatechin gallate (b) deprotonated at the $3^{\prime}-\mathrm{OH}$ position; catechin gallate (c) and epicatechin gallate (d) deprotonated at the 3 "-OH position; catechin gallate (e) and epicatechin gallate (f) deprotonated at the 4 "-OH position; catechin gallate (g) and epicatechin gallate $(\mathbf{h})$ deprotonated at the $5 "-\mathrm{OH}$ position.

occur at the $4^{\prime \prime}-\mathrm{OH}, 5^{\prime \prime}-\mathrm{OH}$, and $3^{\prime}-\mathrm{OH}$ positions. A direct comparison between gas-phase acidities shows that every catechin gallate hydroxyl proton is more acidic than the corresponding epicatechin gallate one. This is particularly noticeable for the $4^{\prime}-\mathrm{OH}$ proton, with a calculated gas-phase acidity of $335.5 \mathrm{kcal} / \mathrm{mol}$ for catechin gallate and $340.9 \mathrm{kcal} / \mathrm{mol}$ for epicatechin gallate.

It is likely, however, that gas-phase conformation is the primary factor contributing to the observed differences in $H / D$ exchange behavior for catechin gallate and epicatechin gallate. Figure 5 presents the lowest energy conformations determined for catechin gallate and epicatechin gallate deprotonated at the $3^{\prime}-\mathrm{OH}$, $3 "-\mathrm{OH}, 4 "-\mathrm{OH}$, and $5 "-\mathrm{OH}$ positions, the most favored 
deprotonation sites according to our calculated gasphase acidities. The catechin gallate molecular ions (Figure 5a, c, e, and g) have the gallate group oriented nearly parallel to the flavonoid B ring in a configuration that is likely to facilitate fast H/D exchange by promoting hydrogen bonding between the charge site and nearby hydroxyl groups and allowing migration of the charge site from the gallate moiety to the $\mathrm{B}$ ring. In contrast, the gallate group is oriented nearly perpendicular to the flavonoid $\mathrm{B}$ ring for the epicatechin gallate molecular ions (Figure 5b, d, f, and h). When the gallate moiety and $\mathrm{B}$ ring adopt such a perpendicular configuration, this is likely to decrease possible hydrogen bonding interactions by increasing the distance between the charge site and other hydroxyl groups, limiting charge site migration between the two groups and slowing the rate of $\mathrm{H} / \mathrm{D}$ exchange.

Our calculated gas-phase acidities may also explain why $\mathrm{H} / \mathrm{D}$ exchange occurs at nonlabile sites only for the nongalloylated catechin stereoisomers. The addition of the gallate moiety substantially increases the acidities of all hydroxyl protons on the galloylated catechins when compared with the nongalloylated stereoisomers. A comparison of the least acidic proton of catechin gallate (at the 7-OH position, $343 \mathrm{kcal} / \mathrm{mol}$ ) and catechin (at the 3-OH position, $370 \mathrm{kcal} / \mathrm{mol}$ ), shows that there is a significant acidity increase with addition of the gallate group, more than $25 \mathrm{kcal} / \mathrm{mol}$ for the least acidic hydrogen. Deuterium incorporation at an allylic or aromatic location requires that the nonlabile hydrogen is in close proximity to the charge site and is relatively acidic compared with labile protons on the molecule, a condition likely to exist for the nongalloylated catechins according to previous computational studies $[65,66]$. For the galloylated catechins, however, the hydroxyl protons are substantially more acidic, decreasing the likelihood that an allylic or aromatic proton would be acidic enough to exchange deuterium by the scrambling mechanism. Addition of the gallate group, therefore, effectively quenches $\mathrm{H} / \mathrm{D}$ exchange via the scrambling mechanism for the catechin stereoisomers.

\section{Conclusions}

Stereoisomerism was found to have little effect on the $\mathrm{H} / \mathrm{D}$ exchange kinetics of the nongalloylated catechins with deuterium oxide. Catechin and epicatechin incorporate deuterium to the same extent with similar rate constants for each deuterium exchange. Gallocatechin and epigallocatechin also exchange the same number of deuteriums with nearly identical individual rate constants. Stereoisomerism was determined to have a considerable impact, however, on the H/D exchange kinetics of the galloylated catechins. Although catechin gallate and epicatechin gallate had the same number of observed exchanges, the magnitude of the individual rate constants differed significantly for the two isomers. Similarly, gallocatechin gallate was determined to have substantially larger individual H/D exchange rate constants than epigallocatechin gallate although both compounds had the same number of observed exchanges. Particularly at longer exchange times, differing deuterium incorporation rates for the galloylated catechins led directly to unique mass spectra for each stereoisomer.

All of the nongalloylated catechin isomers were found to have a greater number of $H / D$ exchanges than predicted by the number of labile hydrogens on the deprotonated molecules, implying that deuterium exchange is occurring via a scrambling mechanism. For the galloylated catechins, however, H/D exchange with deuterium oxide is more likely occurring through a typical relay mechanism, with $S$ chirality of the $\mathrm{C} 2$ carbon (CG, GCG) correlated with faster $\mathrm{H} / \mathrm{D}$ exchange than R (ECG, EGCG).

High-level ab initio calculations were used to predict the probable deprotonation site, low-energy conformations, and gas-phase acidities for the nongalloylated and galloylated catechin isomers to explain our H/D exchange results. For the nongalloylated catechins, stereoisomerism was found to have little effect on calculated gas-phase acidities or the favored deprotonation site. Differences in gas-phase conformation between catechin gallate and epicatechin gallate were used to explain the distinctive H/D exchange kinetics observed for these stereoisomers.

\section{Acknowledgments}

The authors acknowledge financial support for this work generously provided by the Robert A. Welch Foundation (AF-0005) and the Southwestern University faculty sabbatical program. JSB acknowledges funding from the Welch Foundation (F1155) and the National Institutes of Health (R01-GM63512). The authors also thank Nikolaos Bentenitis (Southwestern University) and Joseph Chipuk (University of Texas at Austin) for their advice and guidance while performing the high-level molecular modeling calculations.

\section{References}

1. Schug, K. A.; Lindner, W.; Lemr, K. Isomeric Discrimination of Arginine-Containing Dipeptides Using Electrospray Ionization-Ion Trap Mass Spectrometry and the Kinetic Method. J. Am. Soc. Mass Spectrom. 2004, 15, 840-847.

2. Wu, L.; Meurer, E. C.; Young, B.; Yang, P.; Eberlin, M.; Cooks, G. R. Isomeric Differentiation and Quantification of $\alpha, \beta$-Amino Acid-Containing Tripeptides by the Kinetic Method: Alkali Metal-Bound Dimeric Cluster Ions. Int. J. Mass Spectrom. 2004, 231, 103-111.

3. Reddy, P. N.; Ramesh, V.; Srinivas, R.; Sharma, G. V. M.; Nagendar, P.; Subash, V. Differentiation of Some Positional and Diastereomeric Isomers of Boc-carbo- $\beta^{3}$ Dipeptides Containing Galactose, Xylose, and Mannose Sugars by Electrospray Ionization Tandem Mass Spectrometry (ESI MS/MS). Int. J. Mass Spectrom. 2006, 248, 1115-123.

4. Serafin, S. V.; Maranan, R.; Zhang, K.; Morton, T. H. Mass Spectrometric Differentiation of Linear Peptides Comprised of L-Amino Acids from Isomers Containing One D-Amino Acid Residue. Anal. Chem. 2005, 77, 5480-5487.

5. Brodbelt, J. S.; Zhang, J.; Pikulski, M.; Davis, B. D.; Girennavar, B.; Jayaprakasha, G. K.; Patil, B. S. Characterization of Flavonoids by Tandem Mass Spectrometry and Metal Complexation Strategies. In Potential Benefits of Citrus, Patil, B.S., Ed. ACS Symposium Series, Vol. CMXXXVI; American Chemical Society: Washington DC, 2006; pp 52-69.

6. Pikulski, M.; Aguilar, A.; Brodbelt, J. S. Tunable Transition MetalLigand Complexation for Enhanced Elucidation of Flavonoid Diglyco- 
sides by Electrospray Ionization Mass Spectrometry. J. Am. Soc. Mass Spectrom. 2007, 18, 422-431.

7. Song, F.; Cui, M.; Liu, Z.; Yu, B.; Liu, S. Multiple-Stage Tandem Mass Spectrometry for Differentiation of Isomeric Saponins. Rapid Commun. Mass Spectrom. 2004, 18, 2241-2248.

8. Green, M. K.; Lebrilla, C. B. Ion-Molecule Reactions as Probes of Gas-Phase Structures of Peptides and Proteins. Mass Spectrom. Rev. 1997, $16,53-71$.

9. Zhang, J.; Brodbelt, J. S. Gas-Phase Hydrogen/Deuterium Exchange and Conformations of Deprotonated Flavonoids and Gas-Phase Acidities of Flavonoids. J. Am. Chem. Soc. 2004, 126, 5906-5919.

10. Chipuk, J. E.; Brodbelt, J. S. Gas-Phase Hydrogen/Deuterium Exchange of 5'- and 3'-Mononucleotides in a Quadrupole Ion Trap: Exploring the Role of Conformation and System Energy. J. Am. Soc. Mass Spectrom. 2007, 18, 724-726.

11. Reed, D.; Kass, S. Hydrogen-Deuterium Exchange at Nonlabile Sites: A New Reaction Facet with Broad Implications for Structural and Dynamic Determinations. J. Am. Soc. Mass Spectrom. 2001, 12, 1163-1168.

12. Campbell, S. C.; Rodgers, M. T.; Marzluff, E. M.; Beauchamp, J. L. Deuterium Exchange Reactions as a Probe of Biomolecule Structure. Fundamental Studies of Gas Phase H/D Exchange Reactions of Protonated Glycine Oligomers with $\mathrm{D}_{2} \mathrm{O}, \mathrm{CD}_{3} \mathrm{OD}, \mathrm{CD}_{3} \mathrm{CO}_{2} \mathrm{D}$, and $\mathrm{ND}_{3}$. J. Am. Chem. Soc. 1995, 117, 12840-12854.

13. Kaltashov, I. A.; Doroshenko, V. M.; Cotter, R. J. Gas Phase Hydrogen/ Deuterium Exchange Reactions of Peptide Ions in a Quadrupole Ion Trap Mass Spectrometer. Prot. Struct. Funct. Genet. 1997, 28, 53-58.

14. Reid, G. E.; O'Hair, R. A. J.; Styles, M. L.; McFadyen, W. D.; Simpson, R. J. Gas Phase Ion-Molecule Reactions in a Modified Ion Trap: H/D Exchange of Noncovalent Complexes and Coordinatively Unsaturated Platinum Complexes. Rapid Commun. Mass Spectrom. 1998, 12, 17011708.

15. Reid, G. E.; Simpson, R. J.; O'Hair, R. A. J. Probing the Fragmentation Reactions of Protonated Glycine Oligomers via Multistage Mass Spectrometry and Gas-Phase Ion-Molecule Hydrogen/Deuterium Exchange. Int. J. Mass Spectrom. 1999, 191, 209-230.

16. Schaaff, T. G.; Stephenson, J. L., Jr.; McLuckey, S. A. Gas Phase H/D Exchange Kinetics: DI Versus $\mathrm{D}_{2} \mathrm{O}$. J. Am. Soc. Mass Spectrom. 2000, 11, 167-171.

17. Wyttenbach, T.; Paizs, B.; Barran, P.; Breci, L.; Liu, D.; Suhai, S.; Wysocki, V. H.; Bowers, M. T. The Effect of the Initial Water of Hydration on the Energetics, Structures, and H/D Exchange Mechanism of a Family of Pentapeptides: An Experimental and Theoretical Study. I. Am. Chem. Soc. 2003, 125, 13768-13775.

18. Mao, D.; Babu, K. R.; Chen, Y.-L.; Douglas, D. J. Conformations of Gas-Phase Lysozyme Ions Produced from Two Different Solution Conformations. Anal. Chem. 2003, 75, 1325-1330.

19. Hermann, K.; Wysocki, V.; Vorpagel, E. R. Computational Investigation and Hydrogen/Deuterium Exchange of the Fixed Charge Derivative Tris(2,4,6-Trimethoxyphenyl) Phosphonium: Implications of the Aspartic Acid Cleavage Mechanism. J. Am. Soc. Mass Spectrom. 2005, 16, 1067-1080.

20. Hermann, K. A.; Kuppannan, K.; Wysocki, V. H. Fragmentation of Doubly-Protonated Ion Populations Labeled by H/D Exchange with $\mathrm{CD}_{3}$ OD. Int. J. Mass Spectrom. 2006, 249/250, 93-105

21. Vrkic, A. K.; O'Hair, R. A. J. Gas Phase Reactions of Trimethylborate with the $[\mathrm{M}-\mathrm{H}]^{-}$Ions of Nucleotides and Their Noncovalent Homoand Heterodimer Complexes. Aust. I. Chem. 2003, 56, 389-399.

22. Gard, E.; Green, M. K.; Bregar, J.; Lebrilla, C. B. Gas-Phase Hydrogen/ Deuterium Exchange as a Molecular Probe for the Interaction of Methanol and Protonated Peptides. J. Am. Soc. Mass Spectrom. 1994, 5, 623-631.

23. Wood, T. D.; Chorush, R. A.; Wampler, F. M. III; Little, D. P.; O'Conner, P. B.; McLafferty, F. W. Gas-Phase Folding and Unfolding of Cytochrome $c$ Cations. Proc. Natl. Acad. Sci. U.S.A. 1995, 92, 2451-2454.

24. McLafferty, F. W.; Guan, Z.; Haupts, U.; Wood, T. D.; Kelleher, N. L. Gaseous Conformational Structures of Cytochrome c. J. Am. Chem. Soc. 1998, 120, 4732-4740.

25. Wyttenbach, T.; Bowers, M. T. Gas Phase Conformations of Biological Molecules: The Hydrogen/Deuterium Exchange Mechanism. J. Am. Soc. Mass Spectrom. 1999, 10, 9-14.

26. Jurchen, J. C.; Cooper, R. E.; Williams, E. R. The Role of Acidic Residues and of Sodium Ion Adduction on the Gas-Phase H/D Exchange of Peptides and Peptide Dimers. J. Am. Soc. Mass Spectrom. 2003, 14, 1477-1487.

27. Cox, H. A.; Julian, R. R.; Lee, S. W.; Beauchamp, J. L. Gas-Phase H/D Exchange of Sodiated Glycine Oligomers with $\mathrm{ND}_{3}$ : Exchange Kinetics Do Not Reflect Parent Ion Structures. J. Am. Chem. Soc. 2004, 126, 6485-6490.

28. Rožman, M.; Bertoša, B.; Klasinc, L.; Srzic, D. Gas Phase H/D Exchange of Sodiated Amino Acids: Why Do We See Zwitterions? J. Am. Soc. Mass Spectrom. 2006, 17, 29-36.

29. Grabowski, J. J.; DePuy, C. H.; Van Doren, J. M.; Bierbaum, V. M. Gas-Phase Hydrogen-Deuterium Exchange Reactions of Anions: Kinetics and Detailed Mechanism. I. Am. Chem. Soc. 1985, 107, 7384-7389.

30. Valentine, S. J.; Clemmer, D. E. Hydrogen/Deuterium Exchange Levels of Shape-Resolved Cytochrome $c$ Conformers in the Gas Phase. J. Am. Chem. Soc. 1997, 119, 3558-3566.

31. Madhusudanan, K. P.; Sachdev, K.; Harrison, D. A.; Kulshreshtha, D. K. Gas Phase Hydrogen/Deuterium Exchange Reactions in Chemical
Ionization Mass Spectra of Flavonoids. Int. J. Mass Spectrom. Ion Processes 1984, 62, 289-295.

32. Kondo, K.; Kurihara, M.; Fukuhara, K. Mechanism of Antioxidant Effect of Catechins. In Methods in Enzymology, Vol. CCCXXXV. Flavonoids and Other Polyphenols. Packer L., Eds. Academic Press: San Diego, CA, 2001; pp 203-217.

33. Zhao, B.; Guo, Q. Xin, W. Free Radical Scavenging by Green Tea Polyphenols. In Methods in Enzymology, Vol. CCCXXXV. Flavonoids and Other Polyphenols. Packer L., Eds. Academic Press: San Diego, CA, 2001; pp 217-222.

34. Lambert, J. D. Hong, J.; Yang, G.; Liao, J.; Yang, C. S. Inhibition of Carcinogenesis by Polyphenols: Evidence from Laboratory Investigations. Am. J. Clin. Nutr. 2005, 8 (Suppl), 284S-91S.

35. Demeule, M.; Michaud-Levesque, J.; Annabi, B.; Gingras, D.; Boivin, D. Jodoin, J.; Lamy, S.; Bertrand, Y.; Béliveau, R. Green Tea Catechins as Novel Antitumor and Antiangiogenic Compounds. Curr. Med. Chem. Anti-Cancer. Agents 2002, 2, 441-463.

36. Nie, G.; Wei, T.; Shen, S.; Zhao, B. Polyphenol Protection of DNA Against Damage. In Methods in Enzymology, Vol. CCCXXXV. Flavonoids and Other Polyphenols. Packer L., Eds. Academic Press: San Diego, CA, 2001; pp 232-244.

37. Higdon, J. V.; Frei, B. Tea Catechins and Polyphenols: Health Effects, Metabolism, and Antioxidant Functions. Crit. Rev. Food. Sci. Nutr. 2003, $43,89-143$.

38. Neuhouser, M. L. Dietary Flavonoids and Cancer Risk: Evidence from Human Population Studies. Nutr. Cancer 2004, 50, 1-7.

39. Peters, U.; Poole, C.; Arab, L. Does Tea Affect Cardiovascular Disease? A Meta-Analysis. Am. J. Epidemiol. 2001, 154, 495-503.

40. López-Lázaro, M. Flavonoids as Anticancer Agents: Structure-Activity Relationship Study. Curr. Med. Chem. Anti-Cancer Agents 2002, 2, 691714 .

41. Rice-Evans, C. A.; Miller, N. J. Structure-Antioxidant Activity Relationships of Flavonoids and Isoflavonoids. In Flavonoids in Health and Disease. Rice-Evans, C.; Packer, L., Eds. Marcel Dekker: New York, NY, 1998; pp 199-219.

42. Hollman, P.C.H.; Katan, M.B. Absorption, Metabolism, and Bioavailability of Flavonoids. In Flavonoids in Health and Disease. Rice-Evans C. Packer L., Eds. Marcel Dekker: New York, NY, 1998; pp 483-522.

43. Prasain, J. K.; Wang, C.-C.; Barnes, S. Mass Spectrometric Methods for the Determination of Flavonoids in Biological Samples. Free Rad. Biol. Med. 2004, 37, 1324-1350.

44. Cuyckens, F.; Claeys, M. Mass Spectrometry in the Structural Analysis of Flavonoids. J. Mass Spectrom. 2004, 39, 1-15.

45. Dalluge, J. J.; Nelson, B. C. Determination of Tea Catechins, Review. J. Chromatogr. A 2000, 881, 411-424.

46. Poon, G. C. Analysis of Catechins in Tea Extracts by Liquid Chromatography-Electrospray Ionization Mass Spectrometry. J. Chromatogr. A 1998, 794, 63-74.

47. Lin, Y. Y.; Ng, K. J.; Yang, S. Characterization of Flavonoids by Liquid Chromatography-Tandem Mass Spectrometry. J. Chromatogr. 1993, 629, 389-393.

48. Miketova, P.; Schram, K. H.; Whitney, J.; Li, M.; Huang, R.; Kerns, E. Valcic, S.; Timmerman, B. N.; Rourick, R.; Klohr, S. Tandem Mass Spectrometry Studies of Green Tea Catechins. Identification of Three Minor Components in the Polyphenolic Extract of Green Tea. J. Mass Spectrom. 2000, 35, 860-869.

49. Zhang, K.; Zuo, Y. GC-MS Determination of Flavonoids and Phenolic and Benzoic Acids in Human Plasma after Consumption of Cranberry Juice. J. Agric. Food Chem. 2004, 52, 222-227.

50. Luthria, D. L.; Jones, A. D.; Donovan, J. L.; Waterhouse, A. L. GC-MS Determination of Catechin and Epicatechin Levels in Human Plasma. J. High Res. Chromatogr. 1997, 21, 621-623.

51. Pelillo, M.; Bonoli, M.; Biguzzi, B.; Bendini, A.; Toschi, T. G.; Lercker, G. An Investigation in the Use of HPLC with UV and MS-Electrospray Detection for the Quantification of Tea Catechins. Food Chem. 2004, 87 , 465-470.

52. Miketova, P.; Schram, K. H.; Whitney, J. L.; Kerns, E. H.; Valcic, S.; Timmerman, B. N.; Volk, K. J. Mass Spectrometry of Selected Components of Biological Interest in Green Tea Extracts. J. Nat. Prod. 1998, 61, 461-467.

53. Dalluge, J. J.; Nelson, B. C.; Thomas, J. B.; Welch, M. J.; Sander, L. C. Capillary Liquid Chromatography/Electrospray Mass Spectrometry for the Separation and Detection of Catechins in Green Tea and Human Plasma. Rapid Commun. Mass Spectrom. 1997, 11, 1753-1756.

54. Zeeb, D. J.; Nelson, B. C.; Albert, K.; Dalluge, J. J. Separation and Identification of Twelve Catechins in Tea Using Liquid Chromatography/Atmospheric Pressure Chemical Ionization-Mass Spectrometry. Anal. Chem. 2000, 72, 5020-5026.

55. Dearden, D. V. Kinfit: Kinetics Fitting for Coupled Ordinary Differential Equations, version 2.0; http://chemwww.byu.edu/people/dvdearden/kinfit.htm (posted April 2003).

56. Hehre, W. J. A Guide to Molecular Mechanics and Quantum Chemical Calculations; Wavefunction, Inc.: Irvine, CA, 2003.

57. Frisch, M. J.; Trucks, G. W.; Schlegel, H. B.; Scuseria, G. E.; Robb, M. A.; Cheeseman, J. R.; Montgomery, Jr., J. A.; Vreven, T.; Kudin, K. N. Burant, J. C.; Millam, J. M.; Iyengar, S. S.; Tomasi, J.; Barone, V. Mennucci, B.; Cossi, M.; Scalmani, G.; Rega, N.; Petersson, G.; A.; Nakatsuji, H.; Hada, M.; Ehara, M.; Toyota, K.; Fukuda, R.; Hasegawa, J.; Ishida, M.; Nakajima, T.; Honda, Y.; Kitao, O.; Nakai, H.; Klene, M.; Li, X.; Knox, J. E.; Hratchian, H. P.; Cross, J. B.; Adamo C.; Jaramillo, J.; 
Gomperts, R.; Stratmann, R. E.; Yazyev, O.; Austin, A. J.; Cammi, R.; Pomelli, C.; Ochterski, J. W.; Ayala, P. Y.; Morokuma, K.; Voth, G. A.; Salvador, P.; Dannenberg, J. J.; Zakrzewski, V. G.; Dapprich, S.; Daniels, A. D.; Strain, M. C.; Farkas, O.; Malick, D. K.; Rabuck, A. D.; Raghavachari, K.; Foresman, J. B.; Ortiz, J. V.; Cui, Q. A.; Baboul, G.; Clifford, S.; Cioslowski, J.; Stefanov, B. B.; Liu, G.; Liashenko, A. Piskorz, P.; Komaromi, I.; Martin, R. L.; Fox, D. J.; Keith, T.; Al-Laham, M. A.; Peng, C. Y.; Nanayakkara, A.; Challacombe, M.; Gill, P. M. W.; Johnson, B.; Chen, W.; Wong, M. W.; Gonzalez, C.; Pople, J. A. Gaussian 03, revision C.02; Gaussian Inc: Wallingford, CT, 2004.

58. Scott, A. P.; Radom, L. Harmonic Vibrational Frequencies: an Evaluation of the Hartree-Fock, Moller-Plesset, Quadratic Configuration Interaction, Density Functional Theory, and Semiempirical Scale Factors. J. Phys. Chem. 1996, 100, 16502-16513

59. Irikura, K. K.; Johnson, R. D. III; Kacker, R. N. Uncertainties in Scaling Factors for Ab Initio Vibrational Frequencies. J. Phys. Chem. A 2005, 109, 8430-8437.

60. Martins, H. F. P.; Leal, J. P.; Fernandez, M. T.; Lopes, V. H. C.; Cordeiro, M. N. D. S. Toward the Prediction of Activity of Antioxidants: Experimental and Theoretical Study of the Gas-Phase Acidities of Flavonoids. J. Am. Soc. Mass Spectrom. 2004, 15, 848-861.
61. Leopoldini, M.; Russo, N.; Toscano, M. Gas and Liquid Phase Acidity of Natural Antioxidants. J. Agric. Food Chem. 2006, 54, 3078-85.

62. Rauha, J.-P.; Vuorela, H.; Kostiainen, R. Effect of Eluent on the Ionization Efficiency of Flavonoids by Ion Spray, Atmospheric Pressure Chemical Ionization, and Atmospheric Pressure Photoionization Mass Spectrometry. J. Mass Spectrom. 2001, 36, 1269-1280.

63. de Rijke, E.; Zappey „, H.; Ariese, F.; Gooijer, C.; Brinkman, U. A. T. Liquid Chromatography with Atmospheric Chemical Ionization and Electrospray Ionization Mass Spectrometry of Flavonoids with TripleQuadrupole and Ion-Trap Instruments. J. Chromatogr. A 2003, 984 45-58.

64. Hunt, D. F.; Sethi, S. K. Gas-Phase Ion/Molecule Isotope-Exchange Reactions: Methodology for Counting Hydrogen Atoms in Specific Organic Structural Environments by Chemical Ionization Mass Spectrometry. J. Am. Chem. Soc. 1980, 102, 6953-6963.

65. Zhang, H.-Y.; Wang, L.-F. Are Allylic Hydrogens in Catechins More Extractable than Catecholic Hydrogens? JAOCS 2002, 79, 943-944.

66. Kondo, K.; Kurihara, M.; Miyata, N.; Suzuki, T.; Toyoda, M. Mechanistic Study of Catechins as Antioxidants against Radical Oxidation. Arch. Biochem. Biophys. 1999, 362, 79-86. 